\title{
Genome wide association and linkage analyses identified three loci-4q25, 17q23.2, and 10q11.21-associated with variation in leukocyte telomere length: the Long Life Family Study
}

\author{
Joseph H. Lee ${ }^{1,2,3 * \dagger}$, Rong Cheng ${ }^{1,2+}$, Lawrence S. Honig ${ }^{1,2,4}$, Mary Feitosa ${ }^{5}$, Candace M. Kammerer ${ }^{6,7,8}$, \\ Min S. Kang ${ }^{2}$, Nicole Schupf ${ }^{1,2,3,9}$, Shiow J. Lin ${ }^{5}$, Jason L. Sanders ${ }^{6,8}$, Harold Bae ${ }^{10}$, Todd Druley ${ }^{11}$, \\ Thomas Perls $^{12}$, Kaare Christensen ${ }^{13}$, Michael Province ${ }^{5}$ and Richard Mayeux ${ }^{1,2,3,4,9}$
}

1 Sergievsky Center, College of Physicians and Surgeons, Columbia University, New York, NY, USA

2 Taub Institute, College of Physicians and Surgeons, Columbia University, New York, NY, USA

${ }^{3}$ Department of Epidemiology, School of Public Health, Columbia University, New York, NY, USA

${ }^{4}$ Department of Neurology, College of Physicians and Surgeons, Columbia University, New York, NY, USA

${ }^{5}$ Division of Statistical Genomics, Department of Genetics, Washington University School of Medicine, St. Louis, MO, USA

${ }^{6}$ Department of Epidemiology, University of Pittsburgh, Pittsburgh, PA, USA

7 Department of Human Genetics, University of Pittsburgh, Pittsburgh, PA, USA

${ }^{8}$ Center for Aging and Population Health, University of Pittsburgh, Pittsburgh, PA, USA

${ }^{9}$ Department of Psychiatry, College of Physicians and Surgeons, Columbia University, New York, NY, USA

${ }^{10}$ Department of Biostatistics, Boston University Medical Center, Boston, MA, USA

"Department of Pediatrics and Genetics, Washington University School of Medicine, St. Louis, MO, USA

12 Department of Medicine, Boston University Medical Center, Boston, MA, USA

${ }^{13}$ The Danish Aging Research Center, Epidemiology, University of Southern Denmark, Odense, Denmark

Leanne M. Redman, Pennington

\section{Reviewed by:}

Andrew DeWan, Yale School of

Public Health, USA

Gil Atzmon, Albert Einstein College

of Medicine, USA

*Correspondence:

Joseph H. Lee, Sergievsky Center, Taub Institute, College of Physicians and Surgeons, Columbia University, 630 West 168th Street, P\&S Unit 16, New York, NY 10032, USA e-mail:jh12@columbia.edu

these authors have contributed equally to this work.

\section{Edited by:}

Biomedical Research Center, USA

Leukocyte telomere length is believed to measure cellular aging in humans, and short leukocyte telomere length is associated with increased risks of late onset diseases, including cardiovascular disease, dementia, etc. Many studies have shown that leukocyte telomere length is a heritable trait, and several candidate genes have been identified, including TERT, TERC, OBFC1, and CTC1. Unlike most studies that have focused on genetic causes of chronic diseases such as heart disease and diabetes in relation to leukocyte telomere length, the present study examined the genome to identify variants that may contribute to variation in leukocyte telomere length among families with exceptional longevity. From the genome wide association analysis in 4,289 LLFS participants, we identified a novel intergenic SNP rs7680468 located near PAPSS1 and $D K K 2$ on $4 \mathrm{q} 25$ ( $p=4.7 \mathrm{E}-8)$. From our linkage analysis, we identified two additional novel loci with HLOD scores exceeding three, including 4.77 for 17q23.2, and 4.36 for 10q11.21. These two loci harbor a number of novel candidate genes with SNPs, and our gene-wise association analysis identified multiple genes, including DCAF7, POLG2, CEP95, and SMURF2 at 17q23.2; and RASGEF1A, HNRNPF, ANF487, CSTF2T, and PRKG1 at 10q11.21. Among these genes, multiple SNPs were associated with leukocyte telomere length, but the strongest association was observed with one contiguous haplotype in CEP95 and SMURF2. We also show that three previously reported genes-TERC, MYNN, and $O B F C 1$ - were significantly associated with leukocyte telomere length at $p_{\text {empirical }}<0.05$.

Keywords: telomere length, aging, familial longevity, genome wide association and linkage, family-based study, novel genes

\section{INTRODUCTION}

Telomere shortening is a marker of in vivo cellular aging, and leukocyte telomere length is related to life span (Holt et al., 1996; Chadwick and Cardew, 1997; Shay and Wright, 2001; Aviv et al., 2006; Christensen et al., 2006; Armanios and Blackburn, 2012). Individuals with short leukocyte telomere length are at an increased risk of age-related diseases (e.g., cardiovascular diseases, diabetes, dementia, cancer) and premature subsequent death compared with similarly aged individuals with longer telomeres (Jeanclos et al., 1998; Epel et al., 2004; Aviv, 2009, 2012; Kaplan et al., 2009; Honig et al., 2012; Shaffer et al., 2012; Ye et al., 2013). However, the direction and strength of association between leukocyte telomere length and the risk of these diseases vary across studies (Aviv et al., 2006; Sanders et al., 2012).

Both longevity and leukocyte telomere length are heritable traits with estimates ranging from 20 to 40\% (Perls et al., 1998, 2000, 2007; Cournil and Kirkwood, 2001; Perls and Terry, 2003; Lee et al., 2004; Beekman et al., 2006; Christensen et al., 2006; Hjelmborg et al., 2006; Deelen et al., 2011; Newman et al., 2011; Murabito et al., 2012) and 34-82\% (Aulchenko et al., 2004; 
Vasa-Nicotera et al., 2005; Broer et al., 2013), respectively. As with most complex traits, multiple genetic variants along with environmental and lifestyle factors are likely to contribute to familial longevity. Genome wide linkage studies have identified four chromosomal regions, 14q23.2, 10q26.13, and 3p26.1 (Andrew et al., 2006), and 12p11 (Vasa-Nicotera et al., 2005) that may harbor loci that influence leukocyte telomere length. Genome wide association studies (GWAS), and meta-analysis across several studies in multiple populations have revealed several candidate genes: TERC, telomerase RNA component (Codd et al., 2010), located on 3q26 (Soerensen et al., 2012), and TERT, telomerase reverse transcriptase (5p15.33) (Hartmann et al., 2009; Soerensen et al., 2012). A meta-analysis (Mangino et al., 2012) identified CTC1 (conserved telomere maintenance component 1,17p13.1) and ZNF676 (zinc finger protein 676, 19p12) as candidate genes for telomere homeostasis in humans, and confirmed that minor variants of OBFC1 on 10q24.33 was associated with shorter leukocyte telomere length. Although their function is not certain, these genes appear to be involved in maintenance of chromosome structures.

To identify genetic variants contributing to variation in leukocyte telomere length, we analyzed data from a large cohort of families that had multiple family members who survived to exceptionally old age (the Long Life Family Study). To detect both common and rare variants that contribute to leukocyte telomere length, this study applied two different approaches: family based association analysis and joint linkage and association analysis. In addition, a heterogeneity model for linkage analysis was applied to account for possible genetic heterogeneity across families since different families may achieve longevity through different means.

\section{MATERIALS AND METHODS \\ STUDY DESIGN AND SUBJECTS FOR THE PRIMARY STUDY}

We employed a 2-stage genome wide study in genotyping participants from of the Long Life Family Study (LLFS; http://www. longlifefamilystudy.org). The details of study design and protocols are described by Newman et al. (2011). Briefly, the LLFS is a multicenter study with recruitment from four centers, Boston University Medical Center, Boston, MA, Columbia University Medical Center, New York, NY; University of Pittsburgh, Pittsburgh, PA; and University of Southern Denmark, Odense, Denmark. Nearly 5,000 Caucasian subjects from families with strong evidence for familial longevity had been recruited and examined (Sebastiani et al., 2009). The ascertainment strategy for the Denmark cohort differed slightly from US sites. Individuals who were at least 90 years of age during the study recruitment period were identified in the Danish National Register of Persons (Pedersen et al., 2006). Using the parental information on birth place, names, and parish registers available in regional archives, sibships were identified and, potentially eligible families were identified and contacted to assess the family's eligibility and willingness to participate in the LLFS using the criteria parallel to those used in the US. Of those, 4289 individuals from 586 families had measures of leukocyte telomere length and were included in the genome wide association and linkage analyses.

\section{COVARIATES}

Information on demographic and medical history information was obtained from participants by self-report (Newman et al., 2011). To assess potential confounding effects, multiple covariates were included in a polygenic model of heritability analysis in SOLAR (Almasy and Blangero, 1998, 2010): age, sex, education, site, generation, smoking (ever vs. never), alcohol consumption (yes vs. no), marital status (widowed/divorced vs. never married vs. married), a history of heart disease (yes/no) and diabetes (yes/no), and 20 principle components. In all subsequent analyses, we included covariates that were significant at $p<0.05$ : age, sex, education, site, smoking, alcohol consumption, marital status, a history of heart disease, and principle component 8 (PC8). Even though not all three indicator variables for site were significant, we forced site variables into the model.

\section{MEASUREMENTS OF LEUKOCYTE TELOMERE LENGTH}

Assays of average leukocyte leukocyte telomere length were performed using our modification of a method developed by Cawthon et al. (Cawthon, 2002; Cawthon et al., 2003). Briefly, the coded DNA samples were processed by laboratory personnel, blinded to participant characteristics. Real-time PCR was performed using a CFX384 thermocycler (Biorad, Richmond, CA). Assay method was optimized for use of both telomere (T) and single copy gene (S) amplifications on the same 384well plate, with reference standard DNA samples on each plate. Test DNA samples each underwent two triplicate PCR reactions, with use of "calibrator samples" for correction for inter-plate variability. Amplification primers for telomeres included $\mathrm{T}_{\text {for }}$ : 5' - CGGTTTGTTTGGGTTTGGGTTTGGGTTTGGGTTTGGG TT- $3^{\prime}$ and $\mathrm{T}_{\text {rev }}: 5^{\prime}-$ GGCTTGCCTTACCCTTACCCTTACCCTTA CCCTTACCCT-3', and for single copy gene (beta-globin) $\mathrm{S}_{\text {for }} 5^{\prime}$ - GCTTCTGACACAACTGTGTTCACTAGC- $3^{\prime}$ and $\mathrm{S}_{\mathrm{rev}} 5^{\prime}$ CACCAACTTCATCCACGTTCACC- $3^{\prime}$. Thermocycling parameters were $95^{\circ} \mathrm{C} \times 10 \mathrm{~min}$ activation, followed by 34 cycles of $95^{\circ} \mathrm{C} \times 15 \mathrm{~s}$, and $55^{\circ} \mathrm{C} \times 120 \mathrm{~s}$. Our assay coefficient of variance was $5-8 \%$. T/S ratio was converted to basepairs (bp) leukocyte telomere length by use of the linear regression formula: $b p=$ $(1,585 * \mathrm{~T} / \mathrm{S}$ ratio $)+3582$, obtained by co-analysis of selected DNA samples using both PCR and terminal restriction fragment (non-radioactive TeloTAGGG leukocyte telomere length, Roche Diagnostics, Mannheim, Germany) methods (correlation coefficient $r=0.90)$.

Because of non-normality of the leukocyte telomere length distribution (skewness $=2.22$ and kurtosis $=12.19$ ), we transformed leukocyte telomere length using an inverse normal function to minimize potential inflation of type 1 error rates (Allison et al., 1999; Etzel et al., 2003). Following the transformation, the distribution of leukocyte telomere length conformed to a normal distribution ( kewness $=-0.0078$ and kurtosis $=-0.0489$ ) in all family members (See Figure A1).

\section{GENOTYPING}

SNP Chips manufactured by Illumina (Human Omni $2.5 \mathrm{v} 1$ ) were used by the Center for Inherited Disease Research (CIDR) for genotyping. In depth Quality control (QC)-process was carried out in the Division of Statistical Genomics, Washington 
University in Saint Louis. QC was performed before imputation and included assessment of Mendelian errors as implemented in LOKI (Heath, 1997) and verification of reported pedigree relationships using GRR (Abecasis et al., 2001). 83,774 SNPs with a lower call rate $<98 \%$ per marker were dropped; in addition, a total of 3,647 SNPs with high Mendelian error rate were dropped. Eighteen subjects who did not reach a $97.5 \%$ call rate were dropped. In addition, 153,363 Mendelian errors were set to missing. Of approximately $2.23 \mathrm{M}$ autosomal SNPs, approximately $1.47 \mathrm{M}$ with a minor allele frequency $(\mathrm{MAF})>1 \%$ were used in the analysis.

\section{POPULATION STRUCTURE}

To examine underlying population structure, principal components (PCs) analysis was performed as implemented in Eigenstrat (Patterson et al., 2006; Price et al., 2006). SNPs with MAF <5\%, Hardy-Weinberg equilibrium (HWE) $p$-value $<10^{-6}$, and with missing genotypes were excluded. In addition, 1613 SNPs from some special regions (2q21, 2q21.1, HLA1, and HLA (chromosome 6), 8p23.1, 8p23, 17q21.31, and 17q21.311) were excluded because of known inversions, HLA and other special regions that may drive the principal component (PC) analysis. After QC procedure, a total of 116,867 tag SNPs were used to create PCs model using 1522 unrelated subjects from LLFS and 361 founders from HapMap data (CEPH: Caucasians, Yoruban: YRI- Africans, Asians: Chinese and Japanese, and Tuscans: TSICaucasians) for the same tag SNPs as the ones used for LLFS. The PC model generated 20 PCs, and PC estimators then were expanded, within Eigenstrat framework, to all members of LLFS. PCs were subsequently used as covariates to control for population substructure/admixture.

\section{IMPUTATION}

Imputations were performed based on cosmopolitan phased haplotypes of 1000 Human Genome (1000HG, version 2010-11 data freeze, 2012-03-04 haplotypes; http://www.sph.umich.edu/csg/ abecasis/MaCH/download/1000G.2012-03-14.html). Three programs were used: $\mathrm{MACH}$ for pre-phasing LLFS data (version 1.0.16); MINIMACH for performing imputations (version of May 2012); and ChunkChromosome script for splitting the LLFS data into smaller blocks to speed the process of imputation. In addition a number of SAS programs were implemented to streamline this process as well as transforming the final data into SAS datasets. Imputations were performed in chunks with 5000 SNPs blocks and 1000 SNPs overlap from our data. A number of filters before imputing were implemented in the LLFS genotypic data by removing markers that had MAF $<1 \%$, HWE $p$-value $<10^{-6}$, if LLFS SNPs alleles mismatched with those of $1000 \mathrm{HG}$, and not present in the $1000 \mathrm{HG}$ panel, as well as flipping any SNP when appropriate to the forward strand. A total of $38.05 \mathrm{M}$ SNPs were imputed. For single variant-single trait association with imputed dosage two additional filters were implemented, the MAF $>1 \%$ and the $r^{2}>0.3$ (a quality score from the imputation), which reduced the analysis to $9.25 \mathrm{M}$ variants.

\section{HERITABILITY ANALYSIS}

Heritability was estimated to assess how much phenotypic variance was explained by additive genetic variance using maximum likelihood methods as implemented in SOLAR (Blangero and Almasy, 1997; Almasy and Blangero, 1998). Heritability estimates were computed over all family members within the proband and offspring generations.

\section{FAMILY-BASED ASSOCIATION ANALYSIS}

To determine whether common variants (MAF >1\%) contribute to variation in leukocyte telomere length, a family based genome wide association study (GWAS) was performed. For the GWAS, the most parsimonious linear mixed model was used that comprised several covariates, including age, sex, education, site, smoking, alcohol consumption, marital status, a history of heart disease, and PC8. Of 20 PCs, only PC8 was included in the linear mixed model because it was found to be significant at $p<0.05$ in the multivariate polygenic model described above. This mixed linear model adjusted for relatedness among family members by incorporating the kinship coefficient matrix using the $\mathrm{R}$ functions written by Therneau (Therneau et al., 2012). In addition, to confirm previously reported genes, we examined the regions containing the previously reported genes and applied the linear mixed effects model as above, but we 'shuffled' the phenotype 500 times to obtain empirical p-values at three different levels to correct for multiple testing: SNP-wise, gene-wise, and then experiment wise $p$-values. To obtain gene wise $\mathrm{p}$-values, we shuffled the phenotypes and computed $\mathrm{p}$-values to establish the distribution of smallest $p$ values for 500 replicates, and then counted the number of replicates that exceeded the nominal $p$-values for the SNPs within a gene. To obtain the experiment wise $p$-value, we then extended the approach we used for gene-wise estimation to include all genes.

\section{LINKAGE STUDY}

To determine whether rare variants that contribute to leukocyte telomere length segregate in families, linkage analysis using haplotype based identity-by-descent (IBD: ZAPLO O'Connell, 2000) was performed. Specifically, sets of up to five tightly linked SNPs within $0.5 \mathrm{cM}$ intervals were haplotyped with ZAPLO, generating a set of SNP "super-loci" spaced $\sim 0.5 \mathrm{cM}$ apart and having greater information content than individual SNPs. From these haplotypes, Loki (Heath, 1997) was used to estimate multipoint IBD in intact pedigrees, which was then imported into SOLAR for variance component linkage analysis. Because different quantitative trait loci (QTLs) influencing leukocyte telomere length are likely to segregate in different families (i.e., genetic heterogeneity), admixture (heterogeneity) LOD scores (HLOD) were completed using SOLAR (Blangero et al., 2001, 2013; Almasy and Blangero, 2010). This algorithm computes HLOD using the algorithm by C. A. B Smith in which the null hypothesis of homogeneity is compared to the hypothesis of heterogeneity (Smith, 1961; Ott, 1983). The admixture linkage analysis identified two linkage peaks-17q23.2 and 10q11.21-that had HLOD exceeding 3 when all families were included in the analysis. When restricted to linked families, HLODs increased to 5.86 for $17 \mathrm{q} 23.2$ and 9.69 for $10 q 11.21$ as expected. This finding suggests that subsequent sequencing experiments can be prioritized to include these families. 
To identify the most likely candidate genes from a large set of genes under each linkage peak, family-based Sequence Kernel Association Test (SKAT) was performed to identify genes that are associated with leukocyte telomere length (Wu et al., 2011; Chen et al., 2013). This model adjusts for the same set of covariates as in the linear mixed model and also controls for familial correlation by including kinship coefficient in the model. famSKAT tests whether multiple rare variants contribute to phenotypic variation, and does not assume that all rare variants influence phenotypes in the same direction with the same effect size. Taking one step further, we then applied measured genotype analysis to identify genetic variants that were significantly associated with variation in leukocyte telomere length. In addition, haplotype analysis was conducted when contiguous multiple variants were associated with leukocyte telomere length. Haplotype analysis was performed using PLINK (Purcell et al., 2007) and MERLIN (Abecasis et al., 2002), and the resulting haplotypes from two analyses were identical.

\section{RESULTS}

\section{DESCRIPTIVE STATISTICS}

Of 4289 subjects with telomere assay, 1418 were from the proband generation and the remaining 2871 were from the offspring generation (Table 1). The overall mean age was 70.1 and ranged from 24 to 110 . There was a slightly higher proportion of women compared with men (55.1 vs. $44.9 \%$ ), and the cohort comprised whites primarily. The mean age at blood draw was the youngest for the Danish site and the oldest for the NY sites (67.2 vs. 74.1). When stratified by generation, however, the maximum mean age difference across sites was 1.8 years (range for the proband generation: 89.0-90.8 years; range for the offspring generation: 59.9-61.4 years), pointing to the fact that Danish families have a larger number of offspring. The overall mean years of education was 11.6 years, and differed between cohorts (proband generation $=9.8$ vs. offspring generation $=12.5$ ) .

The mean leukocyte telomere length was $5325.3 \mathrm{bp}(S D=$ 485.5), and the mean leukocyte telomere length was slightly longer for women compared with men ( 5356.7 bp vs. $5287.6 \mathrm{bp}$ ) (Table 2). The mean leukocyte telomere length was shorter in the proband generation compared with the offspring generation (5170.5 bp vs. $5401.8 \mathrm{bp}$, respectively; $p=5.9 \mathrm{E}-50$ ). Leukocyte telomere length was slightly shorter for the Danish cohort compared with the US cohort (5216.2 bp vs. $5366.6 \mathrm{bp}$ ). When analyzed separately, factors that were significantly associated with leukocyte telomere length at $p<0.05$ were heart disease, smoking, drinking and marital status. When these factors were included in the multivariable model in SOLAR as well as SPSS (SPSS, 2013), the following variables remained significant at $p<$ 0.05: site, sex, marital status, education, drinking, smoking, heart disease, and PC8.

\section{HERITABILITY}

The overall heritability of leukocyte telomere length was estimated to be $0.54(S E=0.034)$ in this cohort. Sex specific heritability estimates for men and women were highly significant and similar to each other $\left(h_{\mathrm{men}}^{2}=0.597 \pm 0.064, p=1.80 \mathrm{E}-23\right.$ vs. $\left.h_{\text {women }}^{2}=0.521 \pm 0.053, p=1.17 \mathrm{E}-26\right)$. When restricted to one generation, the heritability estimate for the proband generation was lower than that for offspring generation $\left(h_{\text {proband }}^{2}=0.47\right.$, $S E=0.068$ vs. $\left.h_{\text {offspring }}^{2}=0.848, S E=0.54\right)$.

\section{ALLELIC ASSOCIATION}

Among genotyped SNPs, rs7680468 on chromosome 4q25 reached genome-wide significance $(p=4.7 \mathrm{E}-8)$ in the LLFS dataset (Figure 1). When imputed SNPs were included, two additional SNPs on 4q25-specifically deletion at c4:108229919 and

Table 1 | Demographic and clinical characteristics: restricted to whites.

\begin{tabular}{llllll}
\hline & \multicolumn{1}{c}{ Overall } & \multicolumn{1}{c}{ Boston } & \multicolumn{1}{c}{ Denmark } & New york & Pittsburgh \\
\hline Subjects & 4289 & 1145 & 1178 & 825 & 1141 \\
Age at blood draw (Range) & $70.1 \pm 15.7(24-110)$ & $69.4 \pm 15.9(32-110)$ & $67.2 \pm 14.2(36-104)$ & $74.1 \pm 16.3(24-108)$ & $71.1 \pm 15.9(36-104)$ \\
Proband generation (Range) & $89.5 \pm 6.7(55-110)$ & $89.5 \pm 7.2(55-110)$ & $90.8 \pm 6.3(64-104)$ & $89.2 \pm 6.8(58-108)$ & $89.0 \pm 6.3(71-104)$ \\
Offspring generation (Range) & $60.6 \pm 8.4(24-88)$ & $59.9 \pm 8.2(32-88)$ & $61.4 \pm 8.4(36-87)$ & $60.4 \pm 8.3(24-83)$ & $60.3 \pm 8.5(36-87)$ \\
Age of married-in's (Range) & $64.9 \pm 11.9(24-98)$ & $65.1 \pm 12.6(37-98)$ & $62.6 \pm 10.1(36-94)$ & $68.4 \pm 13.5(24-94)$ & $68.0 \pm 12.8(36-91)$ \\
Proband generation (Range) & $83.0 \pm 7.0(55-98)$ & $83.2 \pm 8.6(55-98)$ & $83.6 \pm 6.6(64-94)$ & $82.2 \pm 6.8(67-94)$ & $83.1 \pm 5.7(71-91)$ \\
Offspring generation (Range) & $61.0 \pm 8.7(24-88)$ & $60.8 \pm 9.1(37-88)$ & $61.0 \pm 8.4(36-87)$ & $60.4 \pm 9.3(24-79)$ & $61.5 \pm 8.8(36-80)$ \\
Men (\%) & $1927(44.9)$ & $509(44.5)$ & $539(45.8)$ & $379(45.9)$ & $500(43.8)$ \\
Education (years) & $11.60 \pm 3.62$ & $12.55 \pm 2.94$ & $9.73 \pm 4.32$ & $12.25 \pm 3.25$ & $12.11 \pm 2.95$ \\
$\%$ Smoking (0 vs. 1 vs. 2)* & $57.1 / 33.4 / 6.9 \%$ & $58.8 / 37.1 / 3.2 \%$ & $50.9 / 31.2 / 14.0 \%$ & $53.8 / 36.0 / 3.9 \%$ & $64.2 / 30.0 / 5.3 \%$ \\
$\%$ Alcohol consumption & $47.4 / 33.2 / 19.2 \%$ & $47.2 / 36.0 / 16.8 \%$ & $26.3 / 36.0 / 37.6 \%$ & $54.9 / 33.9 / 9.8 \%$ & $63.8 / 26.8 / 9.3 \%$ \\
(0 vs. 1 vs. 2)** & & & & & \\
\% Marital status & $21.7 / 12.4 / 65.9 \%$ & $20.2 / 14.4 / 65.4 \%$ & $16.4 / 8.9 / 74.7 \%$ & $27.5 / 13.8 / 58.1 \%$ & $24.2 / 12.8 / 62.8 \%$ \\
(0 vs. 1 vs. 2)*** & & & & $11.30 \%$ & $9.60 \%$ \\
A history of heart disease & $8.60 \%$ & $8.60 \%$ & $5.70 \%$ & & \\
\hline
\end{tabular}

${ }^{*} 0=$ never, $1=$ past, $2=$ current.

${ }^{*} 0=$ never/occasional drinking, $1=1-7$ per week, $2=7$ per week.

${ }^{* *} 0=$ widowed, $1=$ some form of marriage, $2=$ currently married . 
Table 2 | Mean leukocyte telomere length by site and by sex and generation.

\begin{tabular}{|c|c|c|c|c|c|c|c|c|c|c|}
\hline & \multicolumn{2}{|c|}{ Over all } & \multicolumn{2}{|c|}{ Boston } & \multicolumn{2}{|c|}{ Denmark* } & \multicolumn{2}{|c|}{ New york } & \multicolumn{2}{|c|}{ Pittsburgh } \\
\hline \multicolumn{11}{|l|}{ Sex } \\
\hline Men** & 5286.8 & 466.4 & 5359.2 & 518.5 & 5167.8 & 292 & 5294.9 & 504 & 5335.4 & 508.4 \\
\hline Women & 5356.7 & 498.4 & 5370.3 & 490.5 & 5257 & 410.5 & 5420.3 & 584.6 & 5398.5 & 507.4 \\
\hline Offspring & 5401.8 & 505.8 & 5461.4 & 509.7 & 5230.6 & 361.6 & 5500.6 & 622.6 & 5504.1 & 528.8 \\
\hline \multicolumn{11}{|l|}{ Married-in's } \\
\hline Proband & 5165.2 & 370 & 5225.6 & 457.3 & 5023.5 & 221.6 & 5193.4 & 395.6 & 5163.5 & 296.5 \\
\hline Offspring & 5336.6 & 498.5 & 5466.8 & 532.6 & 5217.6 & 432.1 & 5431 & 521.4 & 5440.3 & 539.5 \\
\hline
\end{tabular}

*Mean leukocyte telomere length comparisons differed significantly between Denmark and Boston as well as Denmark and Pittsburgh.

${ }^{*}$ Mean leukocyte telomere length differed between males and females at $p<0.05$ for all sites except for Boston.

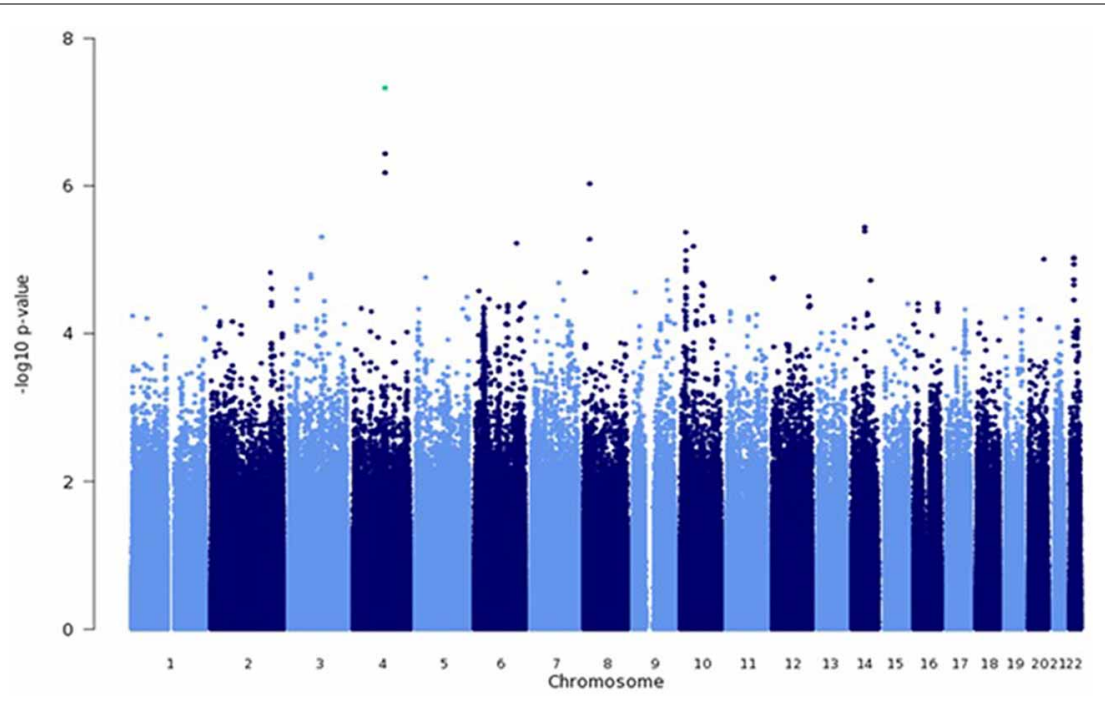

FIGURE 1 | Genome wide association analysis using a mixed linear model. GWAS was performed using a mixed linear model that adjusted for age, sex, education, site, smoking, alcohol consumption, marital status, a history of heart disease, and 1 principle component.

c4:108229924 — were significantly associated with leukocyte telomere length at $p<$ E-8 (Table 3). Altogether five variants, located within or between the DKK2 and PAPSS1 genes, were associated with telomere length at $p<6.6 \mathrm{E}-7$ (Table 3). As these variants span $200 \mathrm{~kb}$, pairwise linkage disequilibrium $\left(\mathrm{D}^{\prime}\right)$ for the first three variants ranged from $\mathrm{D}^{\prime}$ of 0.799 to 0.90 , but $\mathrm{D}^{\prime}$ between SNPs 3 and 4 was 0.25 . In addition, several other SNPs were associated with leukocyte telomere length at suggestive levels of significance ( $p<\mathrm{E}-6)$, including SNPs located near genes candidate genes TMPRSS7 on 3q13.2, TRDMT1 on 10p13, SYT16 on 14q23, TSHZ2 on 20q13.2, and ASCC2 on 22q12.2 (Table 3).

\section{LINKAGE ANALYSIS AND MEASURED GENOTYPE ANALYSIS}

To identify rare variants that segregate in a subset of families that may have been undetected by the linear mixed model, sets of SNP "super-loci" spaced $\sim 0.5 \mathrm{cM}$ apart were used to obtain greater information content than individual SNPs. This linkage analysis based on SOLAR identified four suggestive linkage peaks, including $17 \mathrm{q} 23.2(\mathrm{LOD}=2.52), 10 \mathrm{q} 11.21(\mathrm{LOD}=2.72), 12 \mathrm{p} 12.1$ $(\mathrm{LOD}=1.98)$, and 6q14.1 $(\mathrm{LOD}=1.83)$ (Figure 2). To assess heterogeneity across families, we computed HLODs for these four loci using all families. HLODs were increased to 4.77 for 17q23.2, 4.36 for $10 \mathrm{q} 11.21,2.05$ for $6 \mathrm{q} 14.1$, and 1.99 for $12 \mathrm{p} 12.1$ (LOD = 1.98; Table 4). Thus, we subsequently focused on the two loci with HLOD $>3$ (17q23.2 and 10q11.21), which included multiple candidate genes as shown in Figures 3A,B. To narrow down the number of candidate genes under the linkage peak, a familybased gene-wise study using famSKAT was performed (Wu et al., 2011), yielding four significant candidate genes, namely DCAF7, POLG2, CEP95, and SMURF2 for 17q23.2, and five candidate 
Table 3 | Candidate SNPs from the genome wide association analysis.

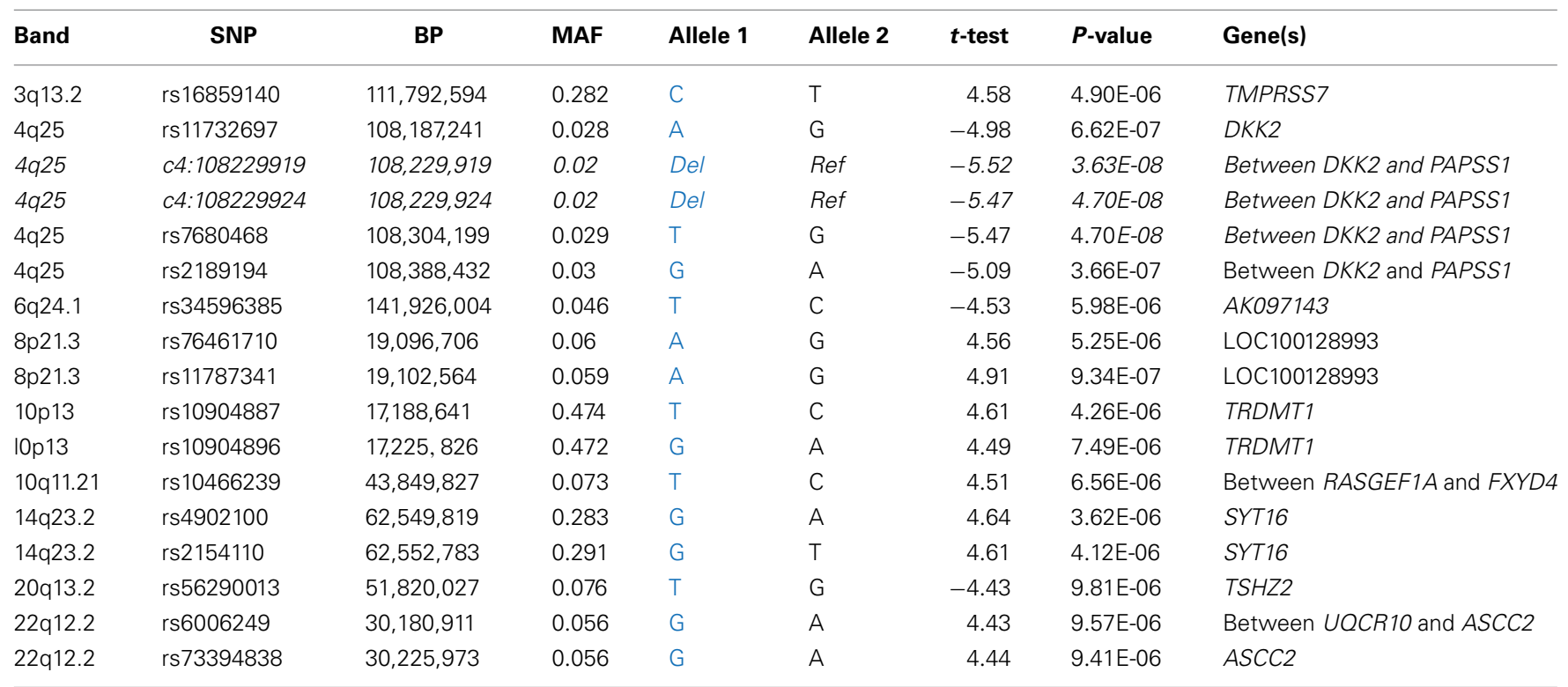

BP based on HG19; imputed SNPS are in italic.

Minor alleles in the LLFS dataset are in blue.

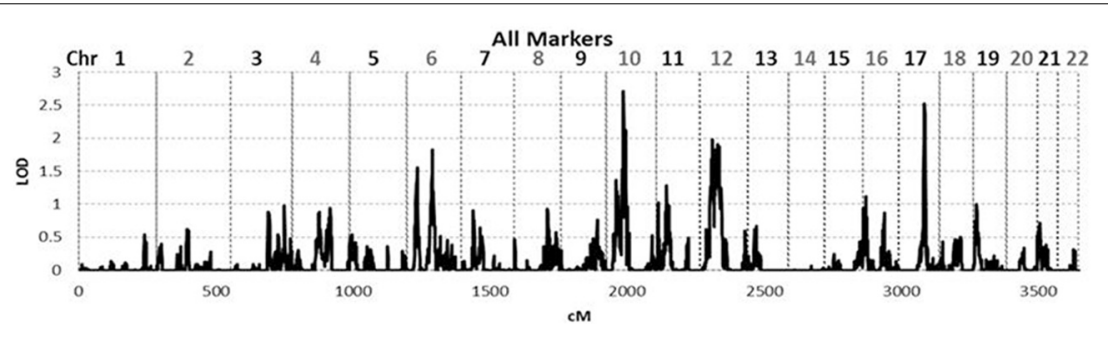

FIGURE 2 | Genome wide linkage analysis using SOLAR. Genome wide linkage analysis based on haplotype IBD, adjusting for age, sex, education, site, smoking, alcohol consumption, marital status, a history of heart disease, and 1 principle component.

genes, namely RASGEF1A, HNRNPF, ANF487, CSTF2T, and PRKG1 for 10q11.21 (Figures 3A,B; Table 4). Two genes-CEP95 $(p=0.000189)$ and SMURF2 $(p=0.000271)$ - had the strongest support for gene-wise association.

To identify variants that may contribute to variation in leukocyte telomere length in these genes, measured genotype analysis was performed for each SNP in the gene using SOLAR (Tables 5A,B). For 17q23.2, multiple SNPs in CEP95 and SMURF2 supported allelic association at $p<\mathrm{E}-5$. We note that the results from the measured genotype analysis using SOLAR did not differ from those in the mixed linear model above. Because multiple contiguous SNP were associated with variation in leukocyte telomere length, haplotype analysis was performed. Table 5A shows that one contiguous haplotype G-T-T-T-G in CEP95 and C-T-G-C-G-C-A-A-C-T in SMURF2 was significantly associated with leukocyte telomere length $(p<0.0057$ for transformed leukocyte telomere length). Due to low allele frequencies of risk variants, two haplotypes-the risk haplotype in black and the reference haplotype in white-were observed as shown in Table 5A. The mean leukocyte telomere length in haplotype carriers was shorter than that in non-carriers ( 5275.5 vs. 5329.4 base pairs, respectively). Similarly, for 10q11.21, a measured genotype analysis identified multiple variants in the HNRNPF gene $(p<$ E-4) and the PRKG1 gene ( $p<\mathrm{E}-5)$.

\section{CONFIRMATION OF PREVIOUS FINDINGS}

Previously implicated genes (TERC, ARPM1, MYNN, OBFC1, and ZNF729) were examined to determine whether families ascertained based exceptional healthy aging also support allelic association for the same five reported SNPs in those genes. As shown in Table 6, 20 SNPs from the five genes were associated with leukocyte telomere length at $p_{\text {nominal }}<0.05$. Of those, TERC, MYNN, and OBFC1 remained significant at gene-wise $p<0.05$ based on a permutation based test, and MYNN was significant at experiment- wise $p<0.05$. However, with the exception of rs1317082 on MYNN, different variants were associated with leukocyte telomere length in the present study.

\section{DISCUSSION}

We identified genetic factors that contribute to variation in leukocyte telomere length in families that were selected for exceptional longevity who also experienced healthy aging. The genome 
Table 4 | Association of leukocyte telomere length and candidate genes under the significant linkage signals.

\begin{tabular}{|c|c|c|c|c|c|c|}
\hline CHR & Gene & $\sim \mathbf{c M}$ & Start (bp) ${ }^{*}$ & end (bp) & $\#$ of SNPs ${ }^{* *}$ & $\mathbf{p}_{\text {gene-wise }}$ \\
\hline & POLG2 & $96.11-96.76$ & $62,466,058$ & $62,493,829$ & 12 & 0.031087 \\
\hline & CEP95 & $96.11-96.76$ & $62,494,043$ & $62,528,641$ & 19 & 0.000189 \\
\hline & SMURF2 & $96.11-96.76$ & $62,536,879$ & $62,694,893$ & 38 & 0.000271 \\
\hline & RASGEF1A & $67.54-68.01$ & $43,682,162$ & $43,859,398$ & 108 & 0.011053 \\
\hline & HNRNPF & $67.54-68.01$ & $43,871,952$ & $43,926,871$ & 36 & 0.032890 \\
\hline & ANF487 & $67.54-68.01$ & $43,904,919$ & $44,050,715$ & 63 & 0.010759 \\
\hline & CSTF2T & $73.00-73.52$ & $53,455,004$ & $53,461,266$ & 16 & 0.046672 \\
\hline
\end{tabular}

${ }^{*}$ HG19.

${ }^{* *}$ Number of genotyped SNPS.

\&HLOD based on all families.

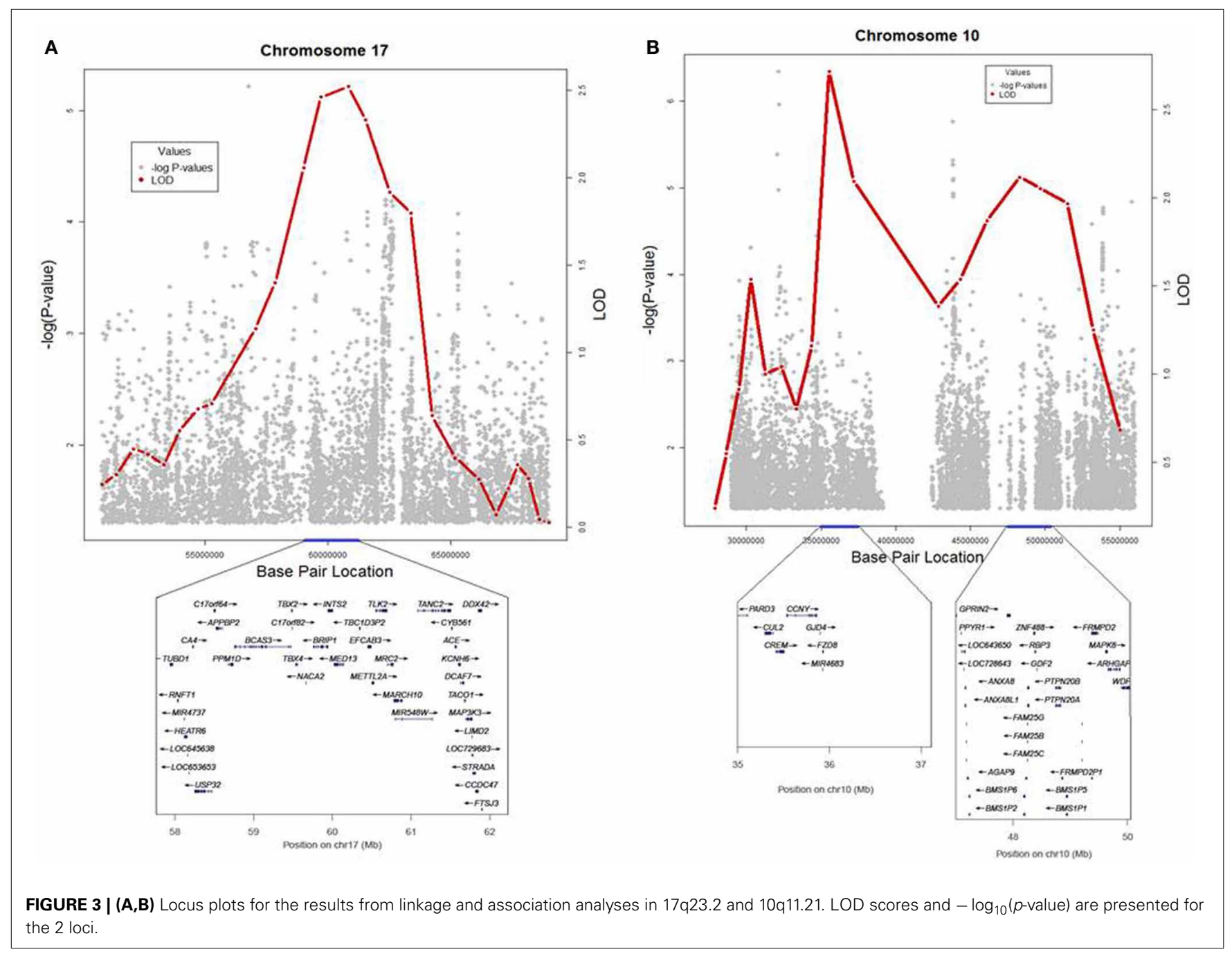


Table 5A | SNP and haplotype association under the 17q23.2 linkage peak.

\begin{tabular}{|c|c|c|c|c|c|c|c|c|c|}
\hline CHR & SNP+ & $\begin{array}{l}\text { Base pair } \\
\text { (HG19) }\end{array}$ & $\mathbf{c M}$ & $\begin{array}{l}\text { Adjusted } \\
\text { multipoint LOD* }\end{array}$ & A1 & A2 & $\begin{array}{c}\text { Measured } \\
\text { genotype } \boldsymbol{p} \text {-value }\end{array}$ & Gene & \\
\hline 17 & rs62075284 & $54,393,314$ & $87.53-88.18$ & 4.82 & $\mathrm{~T}$ & $\mathrm{C}$ & $1.82 \mathrm{E}-04$ & ANKFN1 & intron-variant \\
\hline 17 & rs4968436 & $59,571,053$ & $92.65-93.06$ & 5.26 & C & $\mathrm{T}$ & $1.56 \mathrm{E}-03$ & (TBX4)_beyond & \\
\hline 17 & rs997353 & $60,225,452$ & $93.67-94.28$ & 5.42 & $\mathrm{G}$ & A & 1.01E-03 & (POLRMTP1)_beyond & \\
\hline 17 & rs2429391\& & $60,740,964$ & $94-95$ & & & & & & \\
\hline 17 & rs12949197 & $61,619,379$ & $95.04-95.76$ & 5.86 & $\mathrm{~T}$ & $\mathrm{C}$ & 1.79E-04 & KCNH6 & intron-variant \\
\hline 17 & rs12601211 & $61,637,421$ & $95.04-95.76$ & 5.83 & $A$ & G & 3.86E-04 & DCAF7 & intron-variant \\
\hline 17 & rs1880590 & $62,289,347$ & $95.76-96.11$ & 5.36 & C & $\mathrm{T}$ & 1.72E-04 & TEX2 & intron-variant \\
\hline 17 & rs7359586 & $62,354,992$ & $95.76-96.11$ & 5.30 & C & $\mathrm{T}$ & 1.20E-04 & (TEX2)_beyond & \\
\hline 17 & rs17564034 & $62,358,301$ & $95.76-96.11$ & 5.36 & $\mathrm{~T}$ & G & $6.40 \mathrm{E}-05$ & (TEX2)_beyond & \\
\hline 17 & rs78267724 & $62,512,279$ & $96.11-96.76$ & 5.49 & $\mathrm{~T}$ & $\mathrm{C}$ & 1.06E-04 & CEP95 & intron-variant \\
\hline 17 & rs77203371 & $62,515,094$ & $96.11-96.76$ & 5.49 & $T$ & $\mathrm{C}$ & $9.80 \mathrm{E}-05$ & CEP95 & intron-variant \\
\hline 17 & rs16947846 & $62,516,241$ & $96.11-96.76$ & 5.49 & $G$ & $\mathrm{~T}$ & 1.44E-04 & CEP95 & intron-variant \\
\hline 17 & rs 111573840 & $62,543,431$ & $96.11-96.76$ & 5.49 & C & $T$ & 8.70E-05 & SMURF2 & intron-variant \\
\hline 17 & rs 74375784 & $62,545,042$ & $96.11-96.76$ & 5.49 & $T$ & C & $8.70 \mathrm{E}-05$ & SMURF2 & intron-variant \\
\hline 17 & rs76097728 & $62,547,526$ & $96.11-96.76$ & 5.29 & G & A & 8.90E-05 & SMURF2 & intron-variant \\
\hline 17 & rs16947868 & $62,549,265$ & $96.11-96.76$ & 5.44 & C & $\mathrm{T}$ & 2.87E-04 & SMURF2 & intron-variant \\
\hline 17 & rs77582577 & $62,556,420$ & $96.11-96.76$ & 5.49 & $G$ & $\mathrm{~T}$ & 7.40E-05 & SMURF2 & intron-variant \\
\hline 17 & rs8070258 & $62,574,813$ & $96.11-96.76$ & 5.52 & C & $T$ & $9.20 \mathrm{E}-05$ & SMURF2 & intron-variant \\
\hline 17 & rs17401012 & $62,592,059$ & $96.11-96.76$ & 5.49 & A & C & 8.70E-05 & SMURF2 & intron-variant \\
\hline 17 & rs80319692 & $62,635,137$ & $96.11-96.76$ & 5.49 & A & C & 1.80E-04 & SMURF2 & intron-variant \\
\hline
\end{tabular}

\& The linkage peak was located at rs2429391*-94 cM.

${ }^{*}$ Adjusted LOD at $94 \mathrm{cM}$ in 6 linked families, adjusting for one SNP plus all previously mentioned covariates.

${ }^{*}$ Allelic association for each SNP using all subjects.

Haplotype in CEP95 and SMURF2 are in black.

Rare haplotype frequency for G-T-T-T-G-C-T-G-C-G-C-A-A-C-T in CEP95 and SMURF2 is 0.0377.

wide association analysis revealed that DKK2 and PAPSS1 on $4 \mathrm{q} 25$ are strong candidate genes $(\mathrm{p} 4.7<\mathrm{E}-8)$ that may contribute to variation in leukocyte telomere length, and that several other genes may also be involved. In addition, two novel loci$17 \mathrm{q} 23.2(\mathrm{HLOD}=4.77)$ and 10q11.21 $(\mathrm{HLOD}=4.36)$-had HLOD scores exceeding 3 . From the multiple candidate genes present under the linkage peaks, we identified nine genes that were significantly associated with leukocyte telomere length at gene-wise level, which include four genes in 17q23.2 (specifically DCAF7, POLG2, CEP95, and SMURF2) and five genes in 10q11.21 (specifically, RASGEF1A, HNRNPF, ANF487, CSTF2T, and PRKG1). Among these genes, CEP95 and SMURF2 in 17q23.2 had one contiguous novel haplotype that was significantly associated with leukocyte telomere length. In 10q11.21, SNPs in HNRNPF and PRKG1 were also associated. Further, we confirmed association between leukocyte telomere length and TERC, $M Y N N$, and OBFC1. Our approach of combining association and linkage analyses has identified a set of novel genes that contribute to variation in leukocyte telomere length in families with exceptional longevity characterized by healthy aging. However, it is difficult to decipher the role of these candidate genes in cellular aging since differential cellular aging can arise from the fundamental processes such as cell death, or by influencing the disease processes in age related diseases. Here we discuss several genes that may contribute to biological cellular aging as measured by leukocyte telomere length.

The strongest GWAS signal was observed in a set of five SNPs located in $4 \mathrm{q} 25$, and this finding refines and extends the earlier report of linkage peak at D4S1564 (MLS $=3.65, p=0.044$; $108,376,510-108,376,856 \mathrm{bp})$ based on 137 sibships selected for longevity (Puca et al., 2001). These SNPs are located between the DKK2 and PAPSS1 genes, but one of the SNP localizes to $D K K 2$. Because of limited work done on these genes, their role in cellular aging is unclear. The DKK2 gene is believed to be involved in embryonic development, and interacts with LDLreceptor related protein 6 (LDL6). Based on biological similarity, 
Table 5B | SNP association under the 10q11.21 linkage peak.

\begin{tabular}{|c|c|c|c|c|c|c|c|c|c|}
\hline CHR & SNP & $\begin{array}{l}\text { Base pair } \\
\text { (HG19) }\end{array}$ & $\mathbf{c M}$ & $\begin{array}{l}\text { Adjusted } \\
\text { multipoint LOD* }\end{array}$ & A1 & A2 & $\begin{array}{c}\text { Measured genotype } \\
p \text {-value }\end{array}$ & Gene & \\
\hline 10 & rs117783414 & $29,005,814$ & $56.01-56.52$ & 9.15 & $\mathrm{~T}$ & C & 4.17E-04 & (BAMB/)_beyond & \\
\hline 10 & rs56080575 & $43,788,778$ & $67.54-68.01$ & 9.09 & $A$ & G & $1.52 \mathrm{E}-04$ & (RASGEF1A)_beyond & - \\
\hline 10 & rs2460535 & $43,821,380$ & $67.54-68.01$ & 9.02 & G & $A$ & 4.33E-04 & (FXYD4)_beyond & - \\
\hline 10 & rs59383062 & $43,847,135$ & $67.54-68.01$ & 9.15 & A & $\mathrm{C}$ & 1.46E-04 & (FXYD4)_beyond & - \\
\hline 10 & rs11814409 & $43,852,721$ & $67.54-68.01$ & 9.15 & C & $A$ & 3.39E-04 & (FXYD4)_beyond & - \\
\hline 10 & rs80027918 & $43,881,183$ & $67.54-68.01$ & 9.69 & A & G & $1.91 \mathrm{E}-04$ & HNRNPF & reference \\
\hline 10 & rs13376803 & $43,881,355$ & $67.54-68.01$ & 9.69 & G & $A$ & 2.07E-04 & HNRNPF & reference \\
\hline 10 & rs10409 & $43,881,921$ & $67.54-68.01$ & 9.69 & C & $\mathrm{T}$ & 1.99E-04 & HNRNPF & reference \\
\hline 10 & rs 10899803 & $43,940,898$ & $67.54-68.01$ & 9.69 & & $\mathrm{~T}$ & $1.85 \mathrm{E}-04$ & ZNF487P & intron-variant \\
\hline 10 & rs60830257 & $53,867,534$ & $73.00-73.52$ & 8.45 & A & G & $6.90 \mathrm{E}-05$ & PRKG1 & intron-variant \\
\hline 10 & rs61448551 & $53,874,705$ & $73.00-73.52$ & 8.45 & C & $\mathrm{T}$ & 4.70E-05 & PRKG1 & intron-variant \\
\hline 10 & rs16927026 & $53,887,029$ & $73.00-73.52$ & 8.45 & G & A & $6.50 \mathrm{E}-05$ & PRKG1 & intron-variant \\
\hline 10 & rs58118931 & $53,893,871$ & $73.00-73.52$ & 8.45 & C & $\mathrm{T}$ & 7.10E-05 & PRKG1 & intron-variant \\
\hline
\end{tabular}

\& The 2 linkage peaks ( 63 and 72 cM) were located near these SNPS.

${ }^{*}$ Adjusted mulitipoint LOD at 63 and $72 \mathrm{cM}$ in 12 families, adjusting for one SNP plus all previoiusly adjusted coavariates.

${ }^{* *} p$-values for association for each SNP, adjusting for covariatesGene.

Table 6 | Association in previously reported genes from other studies.

\begin{tabular}{|c|c|c|c|c|c|c|c|c|c|c|c|c|}
\hline Chr & Gene & SNP & Position (bp)* & $N$ & $\begin{array}{l}\text { Allele } \\
\text { freq }\end{array}$ & $\begin{array}{l}\text { Risk } \\
\text { allele }\end{array}$ & Beta & Nominal $\boldsymbol{P}^{* *}$ & SNPwise-P & Genewise- $p$ & $\begin{array}{l}\text { Experiment- } \\
\text { wise-p }\end{array}$ & Role \\
\hline 2 & (CXCR4) & rs75157608 & $136,994,500$ & 4276 & 0.0005 & $\mathrm{~T}$ & -0.96 & 0.04476 & 0.025 & 0.338 & 0.712 & \\
\hline 3 & (TERC) & rs12638862 & $169,477,506$ & 4278 & 0.2498 & G & -0.0714 & 0.00676 & $<0.002$ & 0.010 & 0.374 & \\
\hline 3 & (TERC) & rs12630450 & $169,480,204$ & 4274 & 0.2647 & G & -0.0553 & 0.03325 & 0.02 & 0.060 & 0.864 & \\
\hline 3 & $A R P M 1$ & rs2068178 & $169,485,639$ & 4278 & 0.0219 & $\mathrm{~T}$ & 0.1843 & 0.02198 & 0.038 & 0.082 & 0.852 & missense \\
\hline 3 & $A R P M 1$ & rs9822885 & $169,486,144$ & 4272 & 0.2637 & $\mathrm{G}$ & -0.0565 & 0.03018 & 0.02 & 0.086 & 0.852 & intron-variant \\
\hline 3 & $A R P M 1$ & rs9860874 & $169,486,271$ & 4277 & 0.2634 & $A$ & -0.0579 & 0.02613 & 0.016 & 0.066 & 0.810 & intron-variant \\
\hline 3 & $A R P M 1$ & rs9866776 & $169,487,651$ & 4252 & 0.2619 & $A$ & -0.0571 & 0.0289 & 0.016 & 0.070 & 0.826 & reference \\
\hline 3 & $(M Y N N)$ & rs3821383 & $169,489,946$ & 4263 & 0.2635 & C & -0.0594 & 0.0228 & 0.013 & 0.089 & 0.768 & \\
\hline 3 & MYNN & rs10936599 & $169,492,101$ & 4276 & 0.2289 & $\mathbf{T}$ & -0.0944 & 0.000483 & $<0.002$ & 0.002 & 0.044 & $\begin{array}{l}\text { nc-transcript- } \\
\text { variant }\end{array}$ \\
\hline 3 & MYNN & rs1317082 ${ }^{\&}$ & $169,497,585$ & 4270 & 0.2292 & $\mathbf{G}$ & -0.0964 & 0.000372 & $<0.002$ & 0.002 & 0.037 & intron-variant \\
\hline 10 & (OBFC1) & rs2902638 & $105,636,989$ & 4276 & 0.2572 & C & -0.0604 & 0.0198 & 0.017 & 0.190 & 0.534 & \\
\hline 10 & $O B F C 1$ & rs10748858 & $105,639,514$ & 4277 & 0.4322 & G & 0.0518 & 0.02305 & 0.012 & 0.128 & 0.390 & reference \\
\hline 10 & OBFC1 & rs11191841 & $105,639,611$ & 4276 & 0.4828 & $\mathrm{C}$ & -0.0575 & 0.01105 & $<0.002$ & 0.059 & 0.204 & reference \\
\hline 10 & OBFC1 & rs7100920 & $105,640,978$ & 4277 & 0.4755 & $\mathbf{T}$ & -0.0598 & 0.00806 & $<0.002$ & 0.051 & 0.178 & reference \\
\hline 10 & OBFC1 & rs2067832 & $105,643,134$ & 4268 & 0.4752 & $\mathbf{T}$ & -0.0627 & 0.005524 & $<0.002$ & 0.037 & 0.130 & intron-variant \\
\hline 10 & OBFC1 & rs11598840 & $105,645,181$ & 4270 & 0.1006 & $A$ & 0.0767 & 0.04448 & 0.064 & 0.522 & 0.918 & intron-variant \\
\hline 10 & OBFC1 & rs4918069 & $105,654,391$ & 4274 & 0.2763 & $\mathrm{G}$ & -0.0553 & 0.02839 & 0.018 & 0.256 & 0.638 & intron-variant \\
\hline 10 & OBFC1 & rs77987791 & $105,670,702$ & 4278 & 0.0007 & $\mathrm{~T}$ & 1.111 & 0.0375 & 0.0472 & 0.395 & 0.832 & intron-variant \\
\hline 19 & ZNF729 & rs115647405 & $22,483,912$ & 4278 & 0.0003 & $\mathrm{~T}$ & 2.339 & 0.01871 & 0.01 & 0.224 & 0.532 & intron-variant \\
\hline 19 & ZNF729 & rs76712090 & $22,486,056$ & 4267 & 0.0127 & $\mathrm{~T}$ & -0.2122 & 0.03684 & 0.0312 & 0.405 & 0.823 & intron-variant \\
\hline
\end{tabular}

${ }^{*} H G 19$.

${ }^{* *}$ Nominal p-values are presented for each SNP; however, SNPS with empirical p-value $<0.05$ are shown in bold.

Only the genotyped SNPs are presented.

\& This SNP was previously reported. 
it may be involved in bone diseases, cancer and Alzheimer disease in adults (Magrane and Consortium, 2011). The PAPSS1 gene encodes a trypsinogen that is a member of the trypsin family of serine proteases. Mutations in this gene are associated with hereditary pancreatitis. To date, however, both genes have not been implicated in common diseases in humans.

TRDMT1, Homo sapiens tRNA aspartic acid methyltransferase 1 , on 10p13, is involved in DNA methylation. Because of its role in methylation, Halaschek-Wiener et al. (2009) considered it to be a candidate gene for healthy aging and sequenced 47 individuals who survive to age 85 or older without any major agerelated diseases. This exploratory study observed that TRDMT1 and SIRT3 had the highest frequency of variants; however, the role of TRDMT1 in aging is unclear because allele frequencies for SNPs in this gene were not compared to those in controls. Another candidate gene, SYT16 on 14q23.2 from the GWAS, was reported to be involved in trafficking and exocytosis of secretory vesicles in non-neuronal tissues. Mosing et al. (2010) reported that one of the SNPs in the gene was associated with self-rated health.

Our genome wide linkage analysis revealed several genes that would have been missed by the GWAS, had we restricted our analysis to genes that meet the strict genome wide significance threshold. Genome wide linkage analysis followed by genewise association analysis identified CEP95 (Centrosomal protein $95 \mathrm{kDa}$ ) and SMURF2 (SMAD specific E3 ubiquitin protein ligase 2) as promising candidate genes. SMURF2 is shown to be involved in regulation of neuronal and cell polarity, induction of cellular senescence, and tumor suppression (Blank et al., 2012), suggesting its potential role in cellular aging. On the other hand, very little is known about CEP95. However the shared haplotype encompassing these two genes is a strong candidate region for sequencing. In addition, KCNH6 (potassium voltage-gated channel, subfamily $\mathrm{H}$ (Ether-A-Go-Go-Related), member 6) belongs to a class of voltage-gated ion channels and is believed to be involved in regulating release of neurotransmitters, controlling heart rate, secretion of insulin, neuronal excitability, etc. (http:// genecards.org).

Among several genes on 10q11.21 that showed significant gene-wise association, including RASGEF1A, HNRNPF, ANF487, CSTF2T, and PRKG1, HNRNPF and PRKG1 are candidate genes of interest. HNRNPF is involved in multiple regulatory pathways, and it has been associated with late onset Alzheimer disease, (Grupe et al., 2006) modulate neuronal viability (Boucher et al., 2002), and is also reported to be associated with cancers. PRKG1 regulates cellular platelet activation and adhesion, contraction of smooth muscles, cardiac function, and other processes involved in several functions association with central nervous system function, such as axon guidance, hippocampal and cerebellar learning, etc. These genes may be biologically relevant to healthy aging, requiring further examination.

The present study confirmed the genes that were previously reported to be associated with leukocyte telomere length (Vasa-Nicotera et al., 2005; Andrew et al., 2006; Mangino et al., 2009; Codd et al., 2010; Levy et al., 2010; Mangino et al., 2012). Specifically, we found at least one SNP in TERC, ARPM1, MYNN, OBFC1, and ZNF729 to be nominally associated with variation in leukocyte telomere length; however, TERC, MYNN, and $O B F C 1$ were significantly associated with leukocyte telomere length based on a permutation test at the gene-wise level. Moreover, MYNN was significant at experiment-wise level. For these genes, different variants were significantly associated with leukocyte telomere length, except for MYNN, where rs1317082 ( $p=0.000372$ ) (Mangino et al., 2012) was previously reported.

Several possibilities may explain the differences in findings across studies, including selection, leukocyte telomere length measurements, differences in the distribution of risk factors, etc (Aviv et al., 2006; Christensen et al., 2006; Armanios and Blackburn, 2012; Sanders et al., 2012). First, cellular aging as measured by leukocyte telomere length is likely to be a complex trait. As such, a different set of genetic and environmental risk factors can influence variation in leukocyte telomere length in different cohorts. The LLFS cohort was sampled to recruit families with strong evidence for familial longevity, and this cohort appears to be relatively healthier than other elderly cohorts (Newman et al., 2011). Previously, Newman et al. (2011) showed that compared with other large epidemiologic cohorts (including Cardiovascular Health Study, the Framingham Heart Study, and the New England Centenarian Study), the prevalence of diabetes, chronic pulmonary disease and peripheral artery disease was lower in LLFS family members and the levels of biomarkers (e.g., pulse pressure, triglycerides, HDL, and gate speed) were more favorable. In addition, the heritability estimates for leukocyte telomere length in this cohort ranged from 0.47 for the older proband generation to 0.85 for the relatively younger offspring generation with an overall estimate of 0.54 . These estimates are comparable to those observed in the meta-analysis based on 19,713 subjects by Broer et al. (2013) which observed an overall heritability of 0.70 , with 0.62 for the Netherlands Twin Registry and Queensland Institute Medical Research Twin study and 0.86 for the Leiden Longevity Study. However, given the difference in recruitment for LLFS compared with other studies, it is likely to yield a different set of candidate genes than in other datasets that have been ascertained for familial aggregation of heart disease or a random set of twins, for example. Moreover, there likely to exist substantial differences in life style or the distribution of environmental risk factors in these families selected for familial longevity compared with cohorts recruited based on diseases of interest. With the exception of age, sex, Caucasian ancestry, and atherosclerosis (Sanders and Newman, 2013), the strength and direction of association between leukocyte telomere length and risk factors are equivocal. Thus, to minimize the influence of these risk factors on allelic association, the present study adjusted for potential confounders, including age, sex, education, site, smoking, alcohol consumption, marital status, a history of heart disease, and one principle component because they were significantly associated with leukocyte telomere length in the LLFS dataset. Second, the telomere assays used across studies vary (Sanders and Newman, 2013). This study measured leukocyte telomere length using the quantitative PCR method (T/S ratio) (Cawthon, 2002; Cawthon et al., 2003; Honig et al., 2012), rather than the terminal restriction fragment method (TRF) (Aviv et al., 2011). The impact of methodological difference on the genetic findings is likely to be minimal. Studies that compared these two methods showed that the T/S ratio 
method measures only "canonical" TTAGGG telomere repeats, while the TRF method derives a "telomere" measurement including telomere-adjacent non-canonical sequences. However, over a wide-range, T/S measurements linearly relate to TRF measurements, as shown by many investigators, including Cawthon et al. (2003), Honig et al. (2012), and Aviv et al. (2011). This study supports comparability of the two methods by confirming the previously reported genes. Lastly, because the present study ascertained healthy families selected for familial longevity, there exist very few studies with such extreme sampling exist. Therefore, the present study lacks extensive replication since reported associations vary widely depending on study design (e.g., case-control vs. family based or randomly selected samples vs. extreme samples, etc.).

In sum, the present study identified novel variants in several genes in three loci-4q25,17q23.2, and 10q11.21-that may contribute to variation in leukocyte telomere length in families with exceptional longevity. The findings from this study may facilitate identification of genes that may better explain how cells age, thereby enhancing our understanding of aging mechanisms.

\section{ACKNOWLEDGMENTS}

LLFS was sponsored by the National Institute on Aging (NIA cooperative agreements U01-AG023712, U01-AG23744, U01AG023746, U01-AG023749 and U01-AG023755).

\section{REFERENCES}

Abecasis, G. R., Cherny, S. S., Cookson, W. O., and Cardon, L. R. (2001). GRR: graphical representation of relationship errors. Bioinformatics 17, 742-743. doi: 10.1093/bioinformatics/17.8.742

Abecasis, G. R., Cherny, S. S., Cookson, W. O., and Cardon, L. R. (2002). Merlinrapid analysis of dense genetic maps using sparse gene flow trees. Nat. Genet. 30, 97-101. doi: 10.1038/ng786

Allison, D. B., Neale, M. C., Zannolli, R., Schork, N. J., Amos, C. I., and Blangero, J. (1999). Testing the robustness of the likelihood-ratio test in a variancecomponent quantitative-trait loci-mapping procedure. Am. J. Hum. Genet. 65, 531-544. doi: 10.1086/302487

Almasy, L., and Blangero, J. (1998). Multipoint quantitative-trait linkage analysis in general pedigrees. Am. J. Hum. Genet. 62, 1198-1211. doi: 10.1086/3 01844

Almasy, L., and Blangero, J. (2010). Variance component methods for analysis of complex phenotypes. Cold Spring Harb. Protoc. 2010, pdb top77. doi: 10.1101/pdb.top77

Andrew, T., Aviv, A., Falchi, M., Surdulescu, G. L., Gardner, J. P., Lu, X., et al. (2006). Mapping genetic loci that determine leukocyte telomere length in a large sample of unselected female sibling pairs. Am. J. Hum. Genet. 78, 480-486. doi: $10.1086 / 500052$

Armanios, M., and Blackburn, E. H. (2012). The telomere syndromes. Nat. Rev. Genet. 13, 693-704. doi: 10.1038/nrg3246

Aulchenko, Y. S., Heutink, P., Mackay, I., Bertoli-Avella, A. M., Pullen, J., Vaessen, N., et al. (2004). Linkage disequilibrium in young genetically isolated Dutch population. Eur. J. Hum. Genet. 12, 527-534. doi: 10.1038/sj.ejhg.52 01188

Aviv, A. (2009). Leukocyte telomere length, hypertension, and atherosclerosis: are there potential mechanistic explanations? Hypertension 53, 590-591. doi: 10.1161/HYPERTENSIONAHA.109.128926

Aviv, A. (2012). Genetics of leukocyte telomere length and its role in atherosclerosis. Mutat. Res. 730, 68-74. doi: 10.1016/j.mrfmmm.2011.05.001

Aviv, A., Hunt, S. C., Lin, J., Cao, X., Kimura, M., and Blackburn, E. (2011). Impartial comparative analysis of measurement of leukocyte telomere length/DNA content by Southern blots and qPCR. Nucleic Acids Res. 39, e134. doi: 10.1093/nar/gkr634

Aviv, A., Valdes, A. M., and Spector, T. D. (2006). Human telomere biology: pitfalls of moving from the laboratory to epidemiology. Int. J. Epidemiol. 35, 1424-1429. doi: 10.1093/ije/dyl169
Beekman, M., Blauw, G. J., Houwing-Duistermaat, J. J., Brandt, B. W., Westendorp, R. G., and Slagboom, P. E. (2006). Chromosome 4q25, microsomal transfer protein gene, and human longevity: novel data and a meta-analysis of association studies. J. Gerontol. A Biol. Sci. Med. Sci. 61, 355-362. doi: 10.1093/gerona/61.4.355

Blangero, J., and Almasy, L. (1997). Multipoint oligogenic linkage analysis of quantitative traits. Genet. Epidemiol. 14, 959-964. doi: 10.1002/(SICI)10982272(1997) 14:6<959::AID-GEPI66>3.0.CO;2-K

Blangero, J., Diego, V. P., Dyer, T. D., Almeida, M., Peralta, J., Kent, J. W., et al. (2013). A kernel of truth: statistical advances in polygenic variance component models for complex human pedigrees. Adv. Genet. 81, 1-31. doi: 10.1016/B9780-12-407677-8.00001-4

Blangero, J., Williams, J. T., and Almasy, L. (2001). Variance component methods for detecting complex trait loci. Adv. Genet. 42, 151-181. doi: 10.1016/S00652660(01)42021-9

Blank, M., Tang, Y., Yamashita, M., Burkett, S. S., Cheng, S. Y., and Zhang, Y. E. (2012). A tumor suppressor function of Smurf2 associated with controlling chromatin landscape and genome stability through RNF20. Nat. Med. 18, 227-234. doi: 10.1038/nm.2596

Boucher, S. E., Cypher, M. A., Carlock, L. R., and Skoff, R. P. (2002). Proteolipid protein gene modulates viability and phenotype of neurons. J. Neurosci. 22, 1772-1783.

Broer, L., Codd, V., Nyholt, D. R., Deelen, J., Mangino, M., Willemsen, G., et al. (2013). Meta-analysis of telomere length in 19,713 subjects reveals high heritability, stronger maternal inheritance and a paternal age effect. Eur. J. Hum. Genet. 21, 1163-1168. doi: 10.1038/ejhg.2012.303

Cawthon, R. M. (2002). Telomere measurement by quantitative PCR. Nucleic Acids Res. 30, e47. doi: 10.1093/nar/30.10.e47

Cawthon, R. M., Smith, K. R., O’brien, E., Sivatchenko, A., and Kerber, R. A. (2003). Association between telomere length in blood and mortality in people aged 60 years or older. Lancet 361, 393-395. doi: 10.1016/S0140-6736(03)12 384-7

Chadwick, D., and Cardew, G. (1997). Telomeres and Telomerase. Chichester; New York: John Wiley \& Sons.

Chen, H., Meigs, J. B., and Dupuis, J. (2013). Sequence kernel association test for quantitative traits in family samples. Genet Epidemiol. 37, 196-204. doi: 10.1002/gepi.21703

Christensen, K., Johnson, T. E., and Vaupel, J. W. (2006). The quest for genetic determinants of human longevity: challenges and insights. Nat. Rev. Genet. 7, 436-448. doi: 10.1038/nrg1871

Codd, V., Mangino, M., Van Der Harst, P., Braund, P. S., Kaiser, M., Beveridge, A. J., et al. (2010). Common variants near TERC are associated with mean telomere length. Nat. Genet. 42, 197-199. doi: 10.1038/ng.532

Cournil, A., and Kirkwood, T. B. (2001). If you would live long, choose your parents well. Trends Genet. 17, 233-235. doi: 10.1016/S0168-9525(01)02306-X

Deelen, J., Beekman, M., Uh, H. W., Helmer, Q., Kuningas, M., Christiansen, L., et al. (2011). Genome-wide association study identifies a single major locus contributing to survival into old age; the APOE locus revisited. Aging Cell 10, 686-698. doi: 10.1111/j.1474-9726.2011.00705.x

Epel, E. S., Blackburn, E. H., Lin, J., Dhabhar, F. S., Adler, N. E., Morrow, J. D., et al. (2004). Accelerated telomere shortening in response to life stress. Proc. Natl. Acad. Sci. U.S.A. 101, 17312-17315. doi: 10.1073/pnas.0407162101

Etzel, C. J., Shete, S., Beasley, T. M., Fernandez, J. R., Allison, D. B., and Amos, C. I. (2003). Effect of Box-Cox transformation on power of Haseman-Elston and maximum-likelihood variance components tests to detect quantitative trait Loci. Hum. Hered. 55, 108-116. doi: 10.1159/000072315

Grupe, A., Li, Y., Rowland, C., Nowotny, P., Hinrichs, A. L., Smemo, S., et al. (2006). A scan of chromosome 10 identifies a novel locus showing strong association with late-onset Alzheimer disease. Am. J. Hum. Genet. 78, 78-88. doi: $10.1086 / 498851$

Halaschek-Wiener, J., Amirabbasi-Beik, M., Monfared, N., Pieczyk, M., Sailer, C., Kollar, A., et al. (2009). Genetic variation in healthy oldest-old. PLoS ONE 4:e6641. doi: 10.1371/journal.pone.0006641

Hartmann, N., Reichwald, K., Lechel, A., Graf, M., Kirschner, J., Dorn, A., et al. (2009). Telomeres shorten while Tert expression increases during ageing of the short-lived fish Nothobranchius furzeri. Mech. Ageing Dev. 130, 290-296. doi: 10.1016/j.mad.2009.01.003

Heath, S. C. (1997). Markov chain Monte Carlo segregation and linkage analysis for oligogenic models. Am. J. Hum. Genet. 61, 748-760. doi: 10.1086/515506 
Hjelmborg, J. vB., Iachine, I., Skytthe, A., Vaupel, J. W., Mcgue, M., Koskenvuo, M., et al. (2006). Genetic influence on human lifespan and longevity. Hum. Genet. 119, 312-321. doi: 10.1007/s00439-006-0144-y

Holt, S. E., Shay, J. W., and Wright, W. E. (1996). Refining the telomeretelomerase hypothesis of aging and cancer. Nat. Biotechnol. 14, 836-839. doi: 10.1038/nbt0796-836

Honig, L. S., Kang, M. S., Schupf, N., Lee, J. H., and Mayeux, R. (2012). Association of shorter leukocyte telomere repeat length with dementia and mortality. Arch. Neurol. 69, 1332-1339. doi: 10.1001/archneurol.2012.1541

Jeanclos, E., Krolewski, A., Skurnick, J., Kimura, M., Aviv, H., Warram, J. H., et al. (1998). Shortened telomere length in white blood cells of patients with IDDM. Diabetes 47, 482-486. doi: 10.2337/diabetes.47.3.482

Kaplan, R. C., Fitzpatrick, A. L., Pollak, M. N., Gardner, J. P., Jenny, N. S., Mcginn, A. P., et al. (2009). Insulin-like growth factors and leukocyte telomere length: the cardiovascular health study. J. Gerontol. A Biol. Sci. Med. Sci. 64, 1103-1106. doi: 10.1093/gerona/glp036

Lee, J. H., Flaquer, A., Costa, R., Andrews, H., Cross, P., Lantigua, R., et al. (2004). Genetic influences on life span and survival among elderly African-Americans, Caribbean Hispanics, and Caucasians. Am. J. Med. Genet. A 128A, 159-164. doi: 10.1002/ajmg.a.30062

Levy, D., Neuhausen, S. L., Hunt, S. C., Kimura, M., Hwang, S. J., Chen, W., et al. (2010). Genome-wide association identifies OBFC1 as a locus involved in human leukocyte telomere biology. Proc. Natl. Acad. Sci. U.S.A. 107, 9293-9298. doi: 10.1073/pnas.0911494107

Magrane, M., and Consortium, U. (2011). UniProt Knowledgebase: a hub of integrated protein data. Database (Oxford) 2011, bar009. doi: 10.1093/database/bar009

Mangino, M., Hwang, S. J., Spector, T. D., Hunt, S. C., Kimura, M., Fitzpatrick, A. L., et al. (2012). Genome-wide meta-analysis points to CTC1 and ZNF676 as genes regulating telomere homeostasis in humans. Hum. Mol. Genet. 21, 5385-5394. doi: $10.1093 / \mathrm{hmg} / \mathrm{dds} 382$

Mangino, M., Richards, J. B., Soranzo, N., Zhai, G., Aviv, A., Valdes, A. M., et al. (2009). A genome-wide association study identifies a novel locus on chromosome 18q12.2 influencing white cell telomere length. J. Med. Genet. 46, 451-454. doi: 10.1136/jmg.2008.064956

Mosing, M. A., Verweij, K. J., Medland, S. E., Painter, J., Gordon, S. D., Heath, A. C., et al. (2010). A genome-wide association study of self-rated health. Twin Res. Hum. Genet. 13, 398-403. doi: 10.1375/twin.13.4.398

Murabito, J. M., Yuan, R., and Lunetta, K. L. (2012). The search for longevity and healthy aging genes: insights from epidemiological studies and samples of long-lived individuals. J. Gerontol. A Biol. Sci. Med. Sci. 67, 470-479. doi: 10.1093/gerona/gls089

Newman, A. B., Glynn, N. W., Taylor, C. A., Sebastiani, P., Perls, T. T., Mayeux, R., et al. (2011). Health and function of participants in the Long Life Family Study: a comparison with other cohorts. Aging (Albany. NY). 3, 63-76.

O'Connell, J. R. (2000). Zero-recombinant haplotyping: applications to fine mapping using SNPs. Genet. Epidemiol. 19(Suppl. 1), S64-S70. doi: 10.1002/10982272(2000) 19:1+<::AID-GEPI10>3.0.CO;2-G

Ott, J. (1983). Linkage analysis and family classification under heterogeneity. Ann. Hum. Genet. 47, 311-320. doi: 10.1111/j.1469-1809.1983.tb01001.x

Patterson, N., Price, A. L., and Reich, D. (2006). Population structure and eigenanalysis. PLoS Genet. 2:e190. doi: 10.1371/journal.pgen.0020190

Pedersen, C. B., Gotzsche, H., Moller, J. O., and Mortensen, P. B. (2006). The danish civil registration system. A cohort of eight million persons. Dan. Med. Bull. 53 , 441-449.

Perls, T., Kohler, I. V., Andersen, S., Schoenhofen, E., Pennington, J., Young, R., et al. (2007). Survival of parents and siblings of supercentenarians. J. Gerontol. A Biol. Sci. Med. Sci. 62, 1028-1034. doi: 10.1093/gerona/62.9.1028

Perls, T. T., Bubrick, E., Wager, C. G., Vijg, J., and Kruglyak, L. (1998). Siblings of centenarians live longer. Lancet 351, 1560. doi: 10.1016/S0140-6736(05)61126-9

Perls, T., and Terry, D. (2003). Genetics of exceptional longevity. Exp. Gerontol. 38, 725-730. doi: 10.1016/S0531-5565(03)00098-6

Perls, T., Terry, D. F., Silver, M., Shea, M., Bowen, J., Joyce, E., et al. (2000). Centenarians and the genetics of longevity. Results Probl. Cell Differ. 29, 1-20. doi: 10.1007/978-3-540-48003-7_1

Price, A. L., Patterson, N. J., Plenge, R. M., Weinblatt, M. E., Shadick, N. A., and Reich, D. (2006). Principal components analysis corrects for stratification in genome-wide association studies. Nat. Genet. 38, 904-909. doi: 10.1038/ ng1847
Puca, A. A., Daly, M. J., Brewster, S. J., Matise, T. C., Barrett, J., Shea-Drinkwater, M., et al. (2001). A genome-wide scan for linkage to human exceptional longevity identifies a locus on chromosome 4. Proc. Natl. Acad. Sci. U.S.A. 98, 10505-10508. doi: 10.1073/pnas.181337598

Purcell, S., Neale, B., Todd-Brown, K., Thomas, L., Ferreira, M. A., Bender, D., et al. (2007). PLINK: a tool set for whole-genome association and population-based linkage analyses. Am. J. Hum. Genet. 81, 559-575. doi: 10.1086/519795

Sanders, J. L., Fitzpatrick, A. L., Boudreau, R. M., Arnold, A. M., Aviv, A., Kimura, M., et al. (2012). Leukocyte telomere length is associated with noninvasively measured age-related disease: the cardiovascular health study. J. Gerontol. A Biol. Sci. Med. Sci. 67, 409-416. doi: 10.1093/gerona/glr173

Sanders, J. L., and Newman, A. B. (2013). Telomere length in epidemiology: a biomarker of aging, age-related disease, both, or neither? Epidemiol Rev. 35, 112-131. doi: 10.1093/epirev/mxs008

Sebastiani, P., Hadley, E. C., Province, M., Christensen, K., Rossi, W., Perls, T. T., et al. (2009). A family longevity selection score: ranking sibships by their longevity, size, and availability for study. Am. J. Epidemiol. 170, 1555-1562. doi: 10.1093/aje/kwp309

Shaffer, J. A., Epel, E., Kang, M. S., Ye, S., Schwartz, J. E., Davidson, K. W., et al. (2012). Depressive symptoms are not associated with leukocyte telomere length: findings from the Nova Scotia Health Survey (NSHS95), a population-based study. PLoS ONE 7:e48318. doi: 10.1371/journal.pone.0048318

Shay, J. W., and Wright, W. E. (2001). Ageing and cancer: the telomere and telomerase connection. Novartis Found. Symp. 235, 116-125. discussion: 125-119, 146-119. doi: 10.1002/0470868694.ch11

Smith, C. A. B. (1961). "Homogeneity test for linkage data," in Proceedings From the Second International Congress of Human Genetics (Rome), 6-12.

Soerensen, M., Thinggaard, M., Nygaard, M., Dato, S., Tan, Q., Hjelmborg, J., et al. (2012). Genetic variation in TERT and TERC and human leukocyte telomere length and longevity: a cross-sectional and longitudinal analysis. Aging Cell 11, 223-227. doi: 10.1111/j.1474-9726.2011.00775.x

SPSS, (2013). “IBM SPSS Statistics for Windows.” 21.0 Edn. (Armonk, NY: IBM Corp).

Therneau, T., Atkinson, E., Sinnwell, J., Matsumoto, M., Schaid, D., and Mcdonnell, S. (2012). Package 'kinship2' [Online]. Available online at: http://cran.rproject.org/web/packages/kinship2/kinship2.pdf

Vasa-Nicotera, M., Brouilette, S., Mangino, M., Thompson, J. R., Braund, P., Clemitson, J. R., et al. (2005). Mapping of a major locus that determines telomere length in humans. Am. J. Hum. Genet. 76, 147-151. doi: 10.1086/ 426734

Wu, M. C., Lee, S., Cai, T., Li, Y., Boehnke, M., and Lin, X. (2011). Rare-variant association testing for sequencing data with the sequence kernel association test. Am. J. Hum. Genet. 89, 82-93. doi: 10.1016/j.ajhg.2011.05.029

Ye, S., Shaffer, J. A., Kang, M. S., Harlapur, M., Muntner, P., Epel, E., et al. (2013). Relation between leukocyte telomere length and incident coronary heart disease events (from the 1995 canadian nova scotia health survey). Am. J. Cardiol. 111, 962-967. doi: 10.1016/j.amjcard.2012.12.017

Conflict of Interest Statement: The authors declare that the research was conducted in the absence of any commercial or financial relationships that could be construed as a potential conflict of interest.

Received: 15 September 2013; accepted: 20 December 2013; published online: 17 January 2014.

Citation: Lee JH, Cheng R, Honig LS, Feitosa M, Kammerer CM, Kang MS, SchupfN, Lin SJ, Sanders JL, Bae H, Druley T, Perls T, Christensen K, Province M and Mayeux $R$ (2014) Genome wide association and linkage analyses identified three loci-4q25, 17q23.2, and 10q11.21-associated with variation in leukocyte telomere length: the Long Life Family Study. Front. Genet. 4:310. doi: 10.3389/fgene.2013.00310

This article was submitted to Genetics of Aging, a section of the journal Frontiers in Genetics.

Copyright (c) 2014 Lee, Cheng, Honig, Feitosa, Kammerer, Kang, Schupf, Lin, Sanders, Bae, Druley, Perls, Christensen, Province and Mayeux. This is an openaccess article distributed under the terms of the Creative Commons Attribution License (CC BY). The use, distribution or reproduction in other forums is permitted, provided the original author(s) or licensor are credited and that the original publication in this journal is cited, in accordance with accepted academic practice. No use, distribution or reproduction is permitted which does not comply with these terms. 
Lee et al.

Genomic study of telomere: LLFS

APPENDIX

$$
\lambda=1.015376, \mathrm{SE}=7.8 \mathrm{E}-06: \mathrm{MAF}>0.01
$$

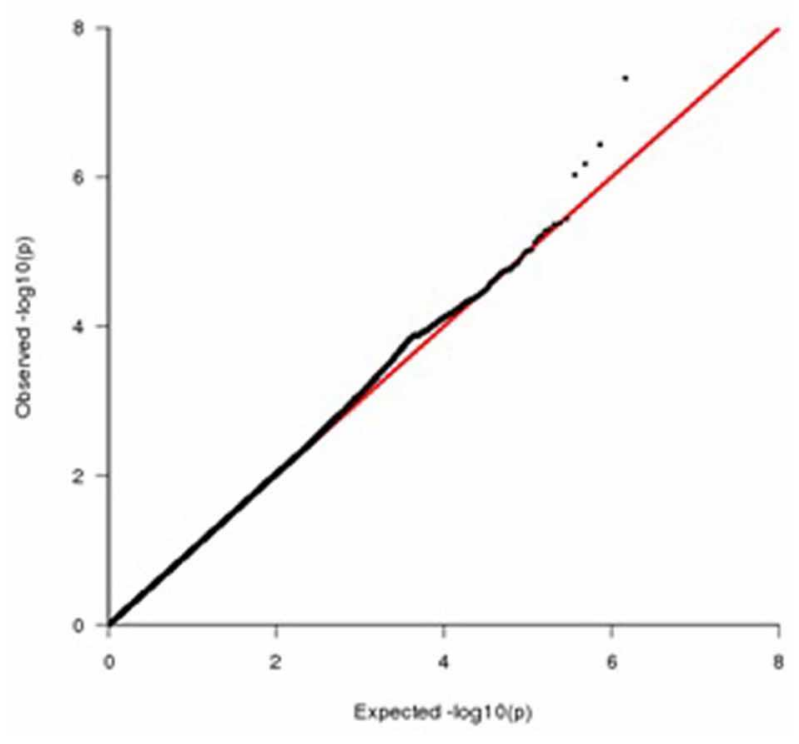

FIGURE A1 | Q-Q Plot of telomere association using Inverse-normal transformation.

www.frontiersin.org

January 2014 | Volume 4 | Article 310 | 13 